\title{
Fibulin-5 localisation in human endometrial cancer shifts from epithelial to stromal with increasing tumour grade, and silencing promotes endometrial epithelial cancer cell proliferation
}

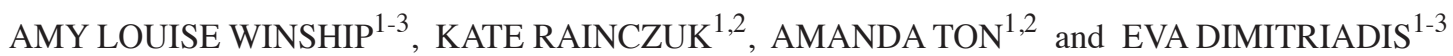 \\ ${ }^{1}$ Centre for Reproductive Health, The Hudson Institute of Medical Research, Melbourne, Victoria 3168; \\ Departments of ${ }^{2}$ Molecular and Translational Medicine and ${ }^{3}$ Anatomy and Developmental Biology, \\ Monash University, Melbourne, Victoria 3800, Australia
}

Received May 20, 2015; Accepted April 15, 2016

DOI: $10.3892 / \mathrm{ol} .2016 .4650$

\begin{abstract}
Endometrial cancer is the most common invasive gynaecological malignancy. While endocrine, genetic and inflammatory factors are thought to contribute to its pathogenesis, its precise etiology and molecular regulators remain poorly understood. Fibulin-5 is an extracellular matrix (ECM) protein that inhibits cell growth and invasion in several cancer cell types and is downregulated in a number of types of human cancer. However, it is unknown whether fibulin-5 plays a role in endometrial tumourigenesis. In the current report, the expression and localisation of fibulin-5 in type I endometrioid human endometrial cancers of grades (G) 1-3 was investigated using reverse transcription-quantitative polymerase chain reaction and immunohistochemistry. Fibulin-5 mRNA was found to be significantly reduced in whole tumour tissues from women across G1-3 compared with benign endometrium $(\mathrm{P}<0.0001)$. Consistently, fibulin-5 protein was also reduced in the tumour epithelial compartment across increasing tumour grades. By contrast, increased protein localisation to the tumour stroma was observed with increasing grade. Knockdown by small interfering RNA in Ishikawa endometrial epithelial cancer cells expressing fibulin-5 stimulated cell adhesion and proliferation in vitro. Fibulin-5 mRNA expression in Ishikawa cells was induced by transforming growth factor- $\beta$ and fibulin- 5 in turn activated extracellular signal-regulated kinases (ERK1/2), suggesting that it may act via the mitogen-activated protein kinase pathway. In summary, the present study identified fibulin-5 as a downregulated ECM gene in human endometrial cancer and observed a shift from epithelial to stromal protein localisation with increasing tumour grade in women.
\end{abstract}

Correspondence to: Professor Eva Dimitriadis, Centre for Reproductive Health, The Hudson Institute of Medical Research, 27-31 Wright Street, Clayton, Melbourne, Victoria 3168, Australia E-mail: evdokia.dimitriadis@hudson.org.au

Key words: fibulin-5, endometrium, endometrioid, gynaecological cancer
These data suggest that loss of fibulin-5 function may promote endometrial cancer progression by enhancing epithelial cell adhesion and proliferation.

\section{Introduction}

Endometrial cancer is the most common invasive gynaecological malignancy in developed countries, with $>280,000$ cases annually (1). There is no effective screening test for early detection and, furthermore, a lack of curative therapies. Alarmingly, the incidence is increasing, particularly in women of reproductive age (2). Mortality is primarily associated with advanced or recurrent disease. Although currently available radio-, brachy- or chemotherapies may achieve a transient treatment response, the median survival time for these women is $<1$ year ( 3 ).

Type I 'endometrioid' carcinoma is the most common type of endometrial cancer, accounting for $\sim 85 \%$ of cases (4). It is often preceded by endometrial hyperplasia, a proliferative process within the endometrial glands that leads to an increase in the glandular-stromal ratio. This process is commonly associated with unopposed estrogen stimulation and may also be due to specific genetic alterations (4). Type I endometrial tumours are staged according to the guidelines of the International Federation of Gynaecology and Obstetrics (FIGO) (5). Tumour grade $(\mathrm{G})$ is based histologically on the extent to which the cancer forms glands that display similar morphology to normal endometrium, and also on metastatic behavior: the extent to which the cancer invades the uterine corpus and the surrounding peritoneum (4). While it is considered that endocrine, genetic and inflammatory factors contribute to its initiation and progression (4), the precise etiology and molecular basis of endometrial cancer are poorly understood. Once the critical regulators are discovered, targeted and more effective treatment options may be developed.

Fibulin-5, also known as DANCE [developmental arteries and neural crest epidermal growth factor (EGF)-like] or EVEC (embryonic vascular EGF-like repeat-containing), is a member of the fibulin family, which is characterised by calcium-binding EGF-like repeats and a globular carboxyl-terminal fibulin-type structure (6). The fibulin family comprises six family members 
(fibulins 1-6), which are commonly expressed extracellular matrix (ECM) proteins localised to the basement membrane, stroma and ECM fibers (7). Functionally, fibulin-5 regulates cell-to-cell and cell-to-matrix communication; it also alters ECM structure and functions in tumourigenesis $(6,8)$. Tumour progression and metastases can depend largely on varying extents of cancer cell proliferative, invasive and/or migratory phenotypes, which also involves the adhesion and de-adhesion of cells.

Fibulin-5 inhibits cancer cell proliferation and invasion in several tumour types (9) and its expression is frequently reduced in a number of types of human cancer, including hepatocellular (10), breast (9), ovarian (9), colon (9), prostate (11), bladder (12) and lung (13) cancers, suggesting a potential tumour suppressor role. However, certain studies have demonstrated a pro-tumourigenic role: Fibulin-5 has been shown to enhance the malignancy of human fibrosarcoma cells (9) and mammary epithelial cells, where it initiates the epithelial-mesenchymal transition (EMT) (14). Therefore, the precise function of fibulin-5 in tumourigenesis differs between different cancer types.

The role of fibulin-5 in human endometrial cancer has never been investigated. We hypothesised that fibulin- 5 expression may be reduced in human endometrial cancer, as in other epithelial malignancies. The present study aimed to determine the expression and localisation of fibulin-5 in human endometrial cancer across G1-3 tumours. Furthermore, the effect of fibulin-5 transient knockdown on Ishikawa endometrial epithelial cancer cell adhesion, proliferation, invasion and migration was determined using the xCELLigence real-time system in vitro.

\section{Materials and methods}

Participants and patient samples. Endometrial cancer tissue biopsies ( $\mathrm{n}=10$ per grade) were collected from postmenopausal women undergoing total abdominal hysterectomy for endometrial carcinoma at the Monash Medical Centre (Melbourne, Australia) (Table I). The Human Ethics Committee approved the research project and informed consent was obtained from each patient. Tumours were graded histologically by a specialist gynaecological pathologist according to the guidelines of FIGO (2009), as described previously (15). Proliferative-phase endometrium $(n=10)$ was collected at curettage from women between days 7 and 13 of their menstrual cycle who were scheduled for tubal ligation as a non-tumour control group. A pathologist declared no obvious endometrial pathology. Women had no steroid treatment or other medication for $\geq 2$ months prior to tissue collection. Written informed consent was obtained from each patient and the study was approved by the Southern Health Human Research and Ethics committee. Biopsies were fixed in $4 \%$ neutral-buffered formalin overnight prior to paraffin embedding.

$R N A$ preparation and reverse transcription-quantitative polymerase chain reaction ( $R T-q P C R)$. RNA from endometrial cancer or benign endometrium whole tissue was obtained from the Victorian Cancer Biobank (Melbourne, Australia). To assess the RNA yield, purity and concentration, $2 \mu 1$ was analysed using a Nanodrop spectrophotometer (ND-1000; Thermo Fisher Scientific, Inc., Wilmington, DE, USA). RT-qPCR was performed as previously described (16). Total RNA (250 ng) was reverse transcribed using Superscript III RNA polymerase, random primers, RNase inhibitor (RNaseOUT), deoxynucleoside triphosphates and First-Strand buffer (Invitrogen; Thermo Fisher Scientific, Inc.) according to the manufacturer's protocol and amplified by PCR. RT-qPCR analyses were performed on the Applied Biosystems 7500HT Fast Block Real Time PCR system (Thermo Fisher Scientific, Inc.) in triplicate (final reaction volume, $10 \mu \mathrm{l}$ ) in 384-well Micro Optical plates with the 2X Fast-Start SYBR Green Master Mix containing ROX passive reference dye (Roche Diagnostics, Indianapolis, IN, USA) and $400 \mathrm{nM}$ primers. The primer sequences were as follows: Fibulin-5 forward, 5'-AGC AGG ATC GAA GGG TTT TT-'3; fibulin-5 reverse, 5'-TGG GTT TGG GAA GAC AGA AC-'3; 18s forward, 5'-GAT CCA TTG GAG GGC AAG TCT-'3; and 18s reverse, 5'-CCA AGA TCC AAC TAC GAG CTT-'3 (Sigma-Aldrich, Castle Hill, Australia). The PCR cycling conditions were as follows: $95^{\circ} \mathrm{C}$ for $10 \mathrm{~min}$; followed by 40 cycles of $95^{\circ} \mathrm{C}$ for $15 \mathrm{sec}$ and $60^{\circ} \mathrm{C}$ for $1 \mathrm{~min}$. Relative expression levels were calculated by the comparative quantification cycle $(\Delta \Delta \mathrm{Cq})$ method (17), with 18 s ribosomal RNA serving as the endogenous control for normalisation. Products were sequenced to confirm specificity.

Immunohistochemistry. Formalin-fixed human endometrial cancer or proliferative-phase endometrial sections $(4 \mu \mathrm{m})$ were dewaxed in Histosol (Chem-Supply, Gillman, Australia; $2 \times 10 \mathrm{~min}$ ) and rehydrated in ethanol, and antigen retrieval was performed in $0.01 \mathrm{M}$ sodium citrate $(\mathrm{pH} 6)$ prior to quenching of endogenous peroxidase activity with $3 \%$ hydrogen peroxide in methanol for $10 \mathrm{~min}$. Non-specific binding was blocked with $10 \%$ normal goat serum and $2 \%$ normal human serum (Sigma-Aldrich, St. Louis, MO, USA), in Tris-buffered saline for $30 \mathrm{~min}$. A polyclonal rabbit anti-human fibulin-5 antibody (\#HPA000848; Sigma-Aldrich; dilution, 1:100) was applied overnight at $4^{\circ} \mathrm{C}$. Negative control isotype rabbit IgG (Dako, Glostrup, Denmark; $0.19 \mu \mathrm{g} / \mathrm{ml}$ ) was included for every tissue section. Antibody localisation was detected by sequential application of biotinylated goat anti-rabbit IgG (\#BA-1000; Vector Laboratories, Inc., Burlingame, CA, USA; dilution, 1:200) for $30 \mathrm{~min}$, followed by the Vectastain Elite ABC kit (\#PK-6100; Vector Laboratories, Inc.) for $30 \mathrm{~min}$. Peroxidase activity was visualised by the application of DakoCytomation diaminobenzidine substrate (Dako). Tissues were counterstained with Harris hematoxylin (Sigma-Aldrich) and mounted. Staining intensities in the epithelial and stromal compartments were scored from 0 (no staining) to 3 (intense staining) by two independent, blinded assessors.

PCR and gel electrophoresis. Total RNA from human endometrial cancer cells [Ishikawa (provided by Dr M. Nishida, Tsukuba University, Tochigi, Japan), HEC1A and AN3CA cells (ATCC, Manassas, VA, USA), representative of human endometrial cancer G1-3, respectively] and primary human proliferative-phase endometrial epithelial cells (which were isolated as described previously) (16), was isolated using the TRI Reagent RNA isolation system (Sigma-Aldrich) following the manufacturer's protocol. All samples were treated with DNase I (Ambion DNAfree; Thermo Fisher Scientific, Inc.) and concentrations were quantified using the NanoDrop 1000. Total 
Table I. Clinical characteristics of the included patients $(n=30)$.

\begin{tabular}{|c|c|c|c|c|}
\hline $\begin{array}{l}\text { Patient } \\
\text { no. }\end{array}$ & $\begin{array}{l}\text { Age, } \\
\text { years }\end{array}$ & $\begin{array}{c}\text { Menopausal } \\
\text { status }\end{array}$ & $\begin{array}{l}\text { Cancer } \\
\text { grade }\end{array}$ & $\% \mathrm{MI}$ \\
\hline 1 & 65 & Post & 1 & 0 \\
\hline 2 & 56 & Post & 1 & 29 \\
\hline 3 & 84 & Post & 1 & 80 \\
\hline 4 & 34 & UK & 1 & 0 \\
\hline 5 & 78 & Post & 1 & 4 \\
\hline 6 & 55 & Post & 1 & 0 \\
\hline 7 & 51 & UK & 1 & 0 \\
\hline 8 & 77 & Post & 1 & 21 \\
\hline 9 & 48 & UK & 1 & 0 \\
\hline 10 & 78 & Post & 1 & 12 \\
\hline 11 & 73 & Post & 2 & 38 \\
\hline 12 & 52 & UK & 2 & UK \\
\hline 13 & 60 & Post & 2 & 18 \\
\hline 14 & 88 & Post & 2 & 73 \\
\hline 15 & 63 & Post & 2 & 100 \\
\hline 16 & 54 & UK & 2 & 38 \\
\hline 17 & 61 & Post & 2 & UK \\
\hline 18 & 60 & Post & 2 & 27 \\
\hline 19 & 75 & Post & 2 & 73 \\
\hline 20 & 71 & Post & 2 & 49 \\
\hline 21 & 54 & Post & 3 & 38 \\
\hline 22 & 59 & Post & 3 & 33 \\
\hline 23 & 77 & Post & 3 & 25 \\
\hline 24 & 59 & UK & 3 & UK \\
\hline 25 & 68 & Post & 3 & 13 \\
\hline 26 & 55 & UK & 3 & 81 \\
\hline 27 & 64 & Post & 3 & 49 \\
\hline 28 & 62 & Post & 3 & 52 \\
\hline 29 & 71 & Post & 3 & 19 \\
\hline 30 & 66 & Post & 3 & UK \\
\hline
\end{tabular}

Post, post-menopausal; UK, unknown; MI, myometrial invasion.

RNA (250 ng) was reverse transcribed using the Superscript III First-Strand Synthesis System (Invitrogen; Thermo Fisher Scientific, Inc.) according to the manufacturer's protocol. PCR was performed using a Veriti Thermal Cycler (Applied Biosystems; Thermo Fisher Scientific, Inc.) and GoTaq Green Master Mix 2X (Promega Corporation, Madison, WI, USA) according to the manufacturer's instructions. cDNA was analysed for fibulin-5 and 18s (sequences as described for RT-qPCR) using reaction conditions as follows: Initial denaturation at $95^{\circ} \mathrm{C}$ for 3 mins; 30 cycles of denaturation at $95^{\circ} \mathrm{C}$ for $30 \mathrm{sec}$, annealing at $60^{\circ} \mathrm{C}$ for $30 \mathrm{sec}$ and extension at $72^{\circ} \mathrm{C}$ for $1 \mathrm{~min}$; and a final extension at $72^{\circ} \mathrm{C}$ for $10 \mathrm{~min}$. The PCR products were run on a $1.5 \%$ agarose gel with an Invitrogen 1,000-bp DNA ladder (Thermo Fisher Scientific, Inc., Carlsbad, CA, USA) to determine gene expression.

Cell culture and cytokine treatments in Ishikawa cells. Ishikawa cells were kindly provided by Dr M. Nishida
(Tsukuba University, Tochigi, Japan) and cultured in Dulbecco's modified Eagle's medium (DMEM) with $10 \%$ foetal calf serum (FCS). Confluent cells were serum starved for $24 \mathrm{~h}$ and treated with recombinant human transforming growth factor- $\beta$ (TGF- $\beta$ ) (\#240-B-010; R\&D Systems, Inc., Minneapolis, MN, USA; 1 or $10 \mathrm{ng} / \mathrm{ml}$ ) or phosphate-buffered saline control. To assess the potential of TGF- $\beta$ to induce fibulin- 5 mRNA expression, cells were collected after $6 \mathrm{~h}$ of treatment, RNA was extracted and RT-qPCR analysis was performed as described. To determine the downstream intracellular target of fibulin-5, confluent cells were serum starved for $24 \mathrm{~h}$ prior to treatment with 0.1 or $0.5 \mu \mathrm{g} / \mathrm{ml}$ recombinant human fibulin-5 (\#3095-FB-025; R\&D Systems, Inc.) (based on pilot studies) for $10 \mathrm{~min}$. Cell lysates were collected using lysis buffer [50 mM Tris-base, $150 \mathrm{mM} \mathrm{NaCl}, 2 \mathrm{mM}$ ethylenediaminetetraacetic acid, $2 \mathrm{mM}$ ethylene glycol tetraacetic acid, $25 \mathrm{mM}$ $\mathrm{NaF}$ and $25 \mathrm{mM} \beta$-glycerolphosphate ( $\mathrm{pH} 7.5)]$ containing protease inhibitor $(2 \mu \mathrm{l} / \mathrm{ml})$ (Thermo Fisher Scientific, Inc.) for western blot.

Small interfering RNA (siRNA) transfection of Ishikawa cells. Ishikawa cells were cultured to $70 \%$ confluence and transfected with commercially generated and validated ON-TARGETplus SMARTpool siRNA (GE Healthcare Dharmacon, Inc., Lafayette, CO, USA) that targeted either fibulin-5 (FBN5 siRNA) or no specific sequence as a scrambled (Scr) control. Delivery was performed using Invitrogen Lipofectamine RNAiMAX Transfection Reagent (Thermo Fisher Scientific, Inc.) according to manufacturer's instructions. Cells were transfected for $72 \mathrm{~h}$ prior to RNA collection to test for transfection efficiency or prior to beginning the functional experiments, as described previously (18).

$x$ CELLigence real-time cell functional studies in Ishikawa cells. Experiments were conducted using the xCELLigence Real-Time Cell Analysis (RTCA) DP instrument (Roche Applied Science; ACEA Biosciences, Inc., San Diego, CA, USA), which was placed in a humidified incubator maintained at $37^{\circ} \mathrm{C}$ with $95 \%$ air $/ 5 \% \mathrm{CO}_{2}$. For adhesion and proliferation, cells were seeded in an E-plate 96 at 10,000 cells/well in 5\% FCS DMEM, and the plate was monitored once every $10 \mathrm{~min}$ for $6 \mathrm{~h}$ (for adhesion), then once every hour for a total of $72 \mathrm{~h}$ (for proliferation). Cell migration and invasion were assessed using CIM-plate 16 (ACEA Biosciences, Inc.) with $8 \mathrm{~mm}$ pores. To measure cell invasion, wells were coated on the upper surface of the transwell with Matrigel (BD Biosciences, Franklin Lakes, NJ, USA; dilution, 1:10). To measure migration, the same protocol was used on an uncoated plate. Cells were seeded into the upper chamber at 10,000/well in 5\% FCS DMEM medium, and 10\% FCS DMEM medium was added to the lower chamber. The CIM-plate 16 was monitored every $30 \mathrm{~min}$ for $72 \mathrm{~h}$ total (18). Data was calculated using RTCA software version 1.2, supplied with the instrument (ACEA Biosciences, Inc.), and exported for statistical analysis.

Sodium dodecyl sulfate-polyacrylamide gel electrophoresis (SDS-PAGE) and western blot analysis. SDS-PAGE and western blot analysis to detect phosphorylated (p) and total extracellular signal-regulated kinases (ERK) 1/2 were performed as previously described (19), using monoclonal 


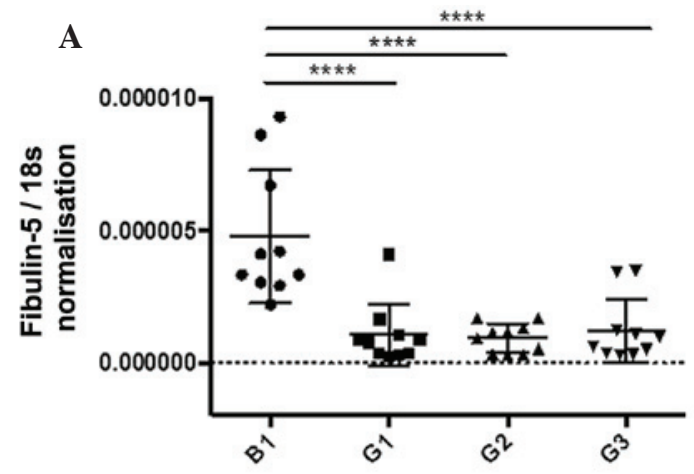

B

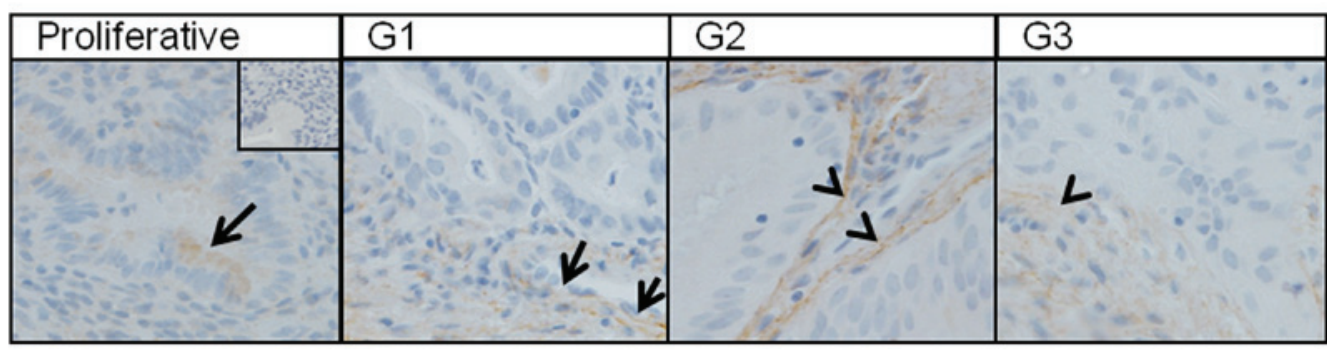

C

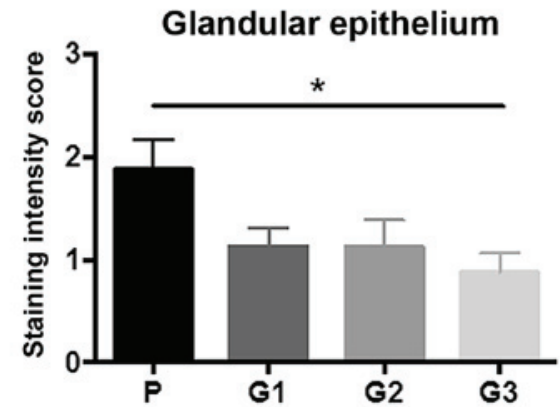

D

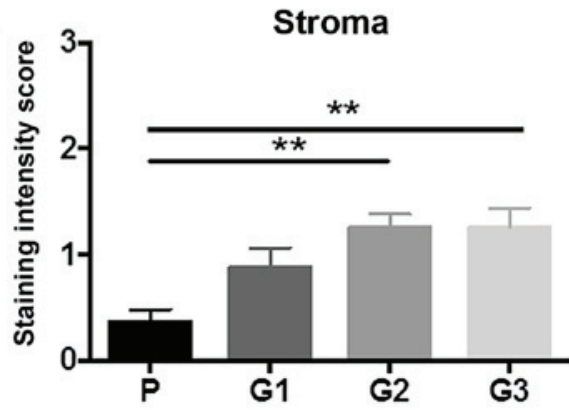

Figure 1. Fibulin-5 mRNA is downregulated in human endometrial cancer but fibulin-5 protein localisation shifts from epithelial to stromal in human endometrial cancer tissue with increasing grade. (A) Endometrial tissue was collected from women with benign endometrium or malignant endometrial cancer of G1, G2 or G3. Fibulin-5 mRNA expression was quantified by reverse transcription-quantitative polymerase chain reaction and normalised to $18 \mathrm{~s}$ (n=10/group). Data are presented as the mean \pm SEM; ${ }^{* * * * *} \mathrm{P}<0.0001$ (one-way ANOVA). (B) Fibulin-5 was immunolocalised in human proliferative-phase endometrium or endometrial cancer G1-3 (n=10/group). Representative photomicrographs are shown at x200 magnification. Arrows denote positive epithelial staining; arrow heads denote positive stromal staining. (C and D) Relative staining intensities are represented as 0 (no staining) to 3 (maximal staining) in (C) the glandular epithelium, or (D) the stroma. Data are presented as the mean \pm SEM; ${ }^{*} \mathrm{P}<0.05$ and ${ }^{* *} \mathrm{P}<0.01$ (one-way ANOVA). B1, benign endometrium; G, grade; $\mathrm{P}$, proliferative-phase endometrium; SEM, standard error of the mean; ANOVA, analysis of variance.

rabbit IgG antibodies against human phospho-p44/42 MAPK (Erk1/2) (Thr202/Tyr204) (\#4370) and p44/42 MAPK (Erk1/2) (\#4695), both purchased from Cell Signaling Technology, Inc. (Danvers, MA, USA)].

Statistical analysis. Statistical analysis was conducted using GraphPad Prism software version 6.0 (GraphPad Software, Inc., La Jolla, CA, USA) and data assessed by Student's $t$-test for two groups. Multiple groups were compared using a one-way analysis of variance with Tukey's post hoc test. $\mathrm{P}<0.05$ was considered to indicate a statistically significant difference.

\section{Results}

Fibulin-5 mRNA and protein are downregulated in human endometrial cancer. RT-qPCR was performed to measure fibulin-5 mRNA levels in whole tissue from G1-3 endometrial tumours compared with benign endometrium. When fibulin-5 expression was normalised to $18 \mathrm{~s}$, a significant reduction in gene expression was observed across all endometrial cancer grades compared to the benign group (all $\mathrm{P}<0.0001$; $\mathrm{n}=10$ /group) (Fig. 1A). Tumour tissues are heterogeneous and gene expression data does not provide information on which cell types produce fibulin-5. Therefore, fibulin-5 was immunolocalised in G1-3 endometrial tumour or proliferative-phase endometrial tissues. Fibulin-5 localised to the glandular epithelium in proliferative-phase endometrium, with little to no staining in the stroma (Fig. 1B). In G1 well-differentiated tumour tissues, fibulin-5 localised to the tumour epithelial and stromal cells, although staining was sporadic throughout the tissue. By comparison in G2 moderately differentiated and G3 poorly differentiated tumours, fibulin-5 almost exclusively localised to the tumour stroma, with some weak staining in the epithelial compartment. When staining intensity was scored, there was a significant reduction in epithelial staining between proliferative endometrium and G3 tumours ( $\mathrm{P}=0.0121 ; \mathrm{n}=10 /$ group) (Fig. $1 \mathrm{C}$ ) and a significant increase in staining in the tumour stroma in $\mathrm{G} 2$ and $\mathrm{G} 3$ compared to proliferative phase endometrium $(\mathrm{P}=0.0010$ and $\mathrm{P}=0.0060$, respectively; $n=10 /$ group) (Fig. 1D). 
A

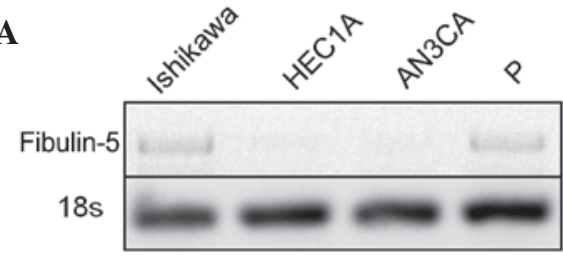

B

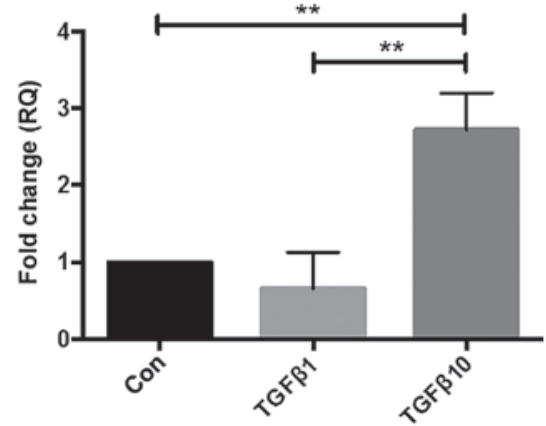

Figure 2. Fibulin-5 mRNA expression and regulation by TGF- $\beta$ in human endometrial cancer cells. (A) Fibulin-5 mRNA expression was determined by PCR in the human endometrial cancer cell lines Ishikawa, HEC1A and AN3CA (representative of G1-3 cancers, respectively), compared with isolated epithelial cells from primary human proliferative-phase endometrium. 18 s was used as a loading control. (B) Ishikawa cells were treated with 1 or $10 \mathrm{ng} / \mathrm{ml} \mathrm{TGF}-\beta$ for $6 \mathrm{~h}$ and fibulin- $5 \mathrm{mRNA}$ expression analysed by reverse transcription-quantitative PCR, normalised to $18 \mathrm{~s}$. Data are presented as the mean \pm standard error of the mean; ${ }^{* *} \mathrm{P}<0.01 ; \mathrm{n}=3$ experiments. TGF- $\beta$, transforming growth factor $\beta$; PCR, polymerase chain reaction; $\mathrm{P}$, proliferative-phase endometrium; Con, control; RQ, relative quantitation.

Fibulin-5 mRNA is downregulated in endometrial cancer cell lines with increasing grade. To perform functional studies, the gene expression of fibulin-5 in human endometrial cancer cell lines was determined. G1-derived Ishikawa cells and proliferative-phase endometrial epithelial cells expressed fibulin-5, whilst G2-derived HEC1A and G3-derived AN3CA cells did not (Fig. 2A). Given this, Ishikawa cells were used for all functional studies.

TGF- $\beta$ induces fibulin-5 expression in Ishikawa endometrial epithelial cancer cells. To investigate the regulation of fibulin-5 in human endometrial epithelial cancer cells, Ishikawa cells were treated with TGF- $\beta$, which is associated with EMT and known to regulate fibulin-5 in other tumour types (14). A significant 2.8 -fold increase in fibulin-5 gene expression following treatment with $10 \mathrm{ng} / \mathrm{ml}$ TGF- $\beta$ was observed compared to the control and the lower concentration of TGF- $\beta(1 \mathrm{ng} / \mathrm{ml})$ after $6 \mathrm{~h}(\mathrm{P}=0.0062$ and $\mathrm{P}=0.0074$, respectively; $\mathrm{n}=3$ /group) (Fig. 2B).

Fibulin-5 siRNA knockdown increases Ishikawa cell adhesion and proliferation. Ishikawa cells were the only cell line tested that expressed fibulin-5. To mimic the loss of fibulin-5 gene expression observed in endometrial cancer in women, fibulin-5 gene expression was transiently silenced using siRNA. This led to a significant $77 \%$ reduction in transcript levels in FBN5 siRNA-treated Ishikawa cells compared to the Scr control ( $\mathrm{P}=0.0037 ; \mathrm{n}=3$ /group) (Fig. 3A). Subsequently, the adhesion, proliferation, invasion and migration functions of Scr- or FBN5 siRNA-treated Ishikawa cells were tested on the xCELLigence RTCA system. At 6 h, FBN5 siRNA-treated Ishikawa cells
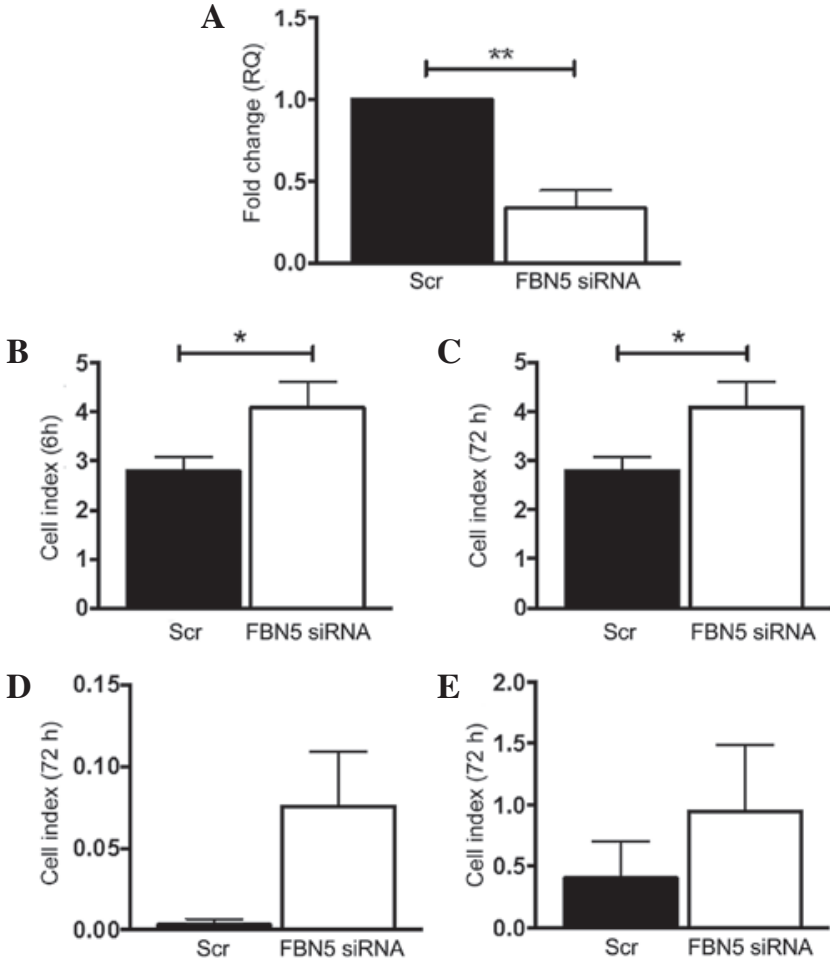

Figure 3. Fibulin-5 silencing by siRNA alters endometrial cancer cell function (A) Knockdown of fibulin-5 was confirmed by reverse transcription-quantitative polymerase chain reaction, normalised to $18 \mathrm{~s}$, expressed as fold-change (RQ) from the control. The effects of Scr control or FBN5 siRNA treatment on Ishikawa cell (B) adhesion, (C) proliferation, (D) invasion and (E) migration were determined using the xCELLigence Real-Time Cell Analysis system, which measures electrical impedance caused by cell attachment and spreading, expressed as the Cell Index. Experiments were repeated in three cell passages in triplicate wells. Data are presented as the mean \pm standard error of the mean. ${ }^{*} \mathrm{P}<0.05$ and ${ }^{* *} \mathrm{P}<0.01$ (Students $t$-test); $\mathrm{n}=3$ /group. siRNA, small interfering RNA; RQ, relative quantitation; Scr, scrambled sequence; FBN5, fibulin-5.
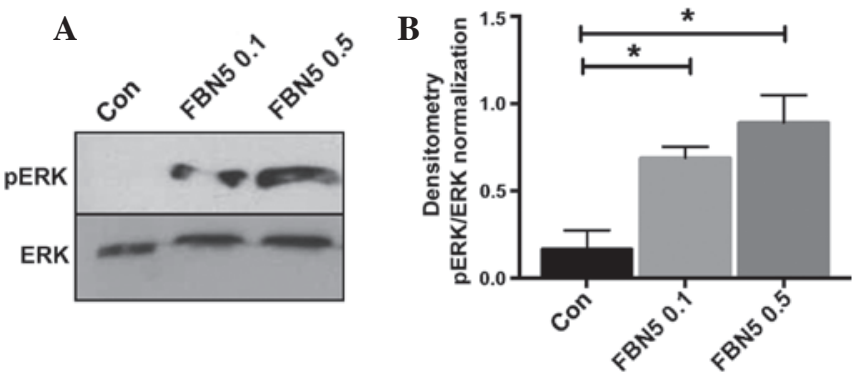

Figure 4. Fibulin-5 activates ERK in Ishikawa cells. (A) Ishikawa cells were treated with phosphate-buffered saline vehicle control (Con), or with 0.1 or $0.5 \mu \mathrm{g} / \mathrm{ml}$ recombinant human fibulin-5 (FBN5 0.1 and FNB5 0.5, respectively) and cell lysates collected after $10 \mathrm{~min}$. Phosphorylated (p)ERK was detected by western blotting and total ERK was used as a loading control. (B) Densitometry was performed on $\mathrm{n}=2$ blots at $10 \mathrm{~min}$ and $\mathrm{pERK}$ was normalised to ERK. Data are presented as the mean \pm standard error of the mean. ${ }^{*} \mathrm{P}<0.05$. ERK, extracellular signal-regulated kinase.

adhered significantly more than the $\mathrm{Scr}$ control group $(\mathrm{P}=0.0475$; $\mathrm{n}=3$ /group) (Fig. 3B), and by $72 \mathrm{~h}$, there was a significant increase in FBN5 siRNA-treated Ishikawa cell proliferation vs. the Scr control ( $\mathrm{P}=0.0384 ; \mathrm{n}=3$ /group) (Fig. 3C). At $72 \mathrm{~h}$ there was an increasing trend in both invasion and migration in FBN5 siRNA-treated cells compared to the control group $(\mathrm{P}=0.0936$ and $\mathrm{P}=0.1205$, respectively) (Fig. 3D and $\mathrm{E}$ ). 
Fibulin-5 activates ERK in Ishikawa cells. To determine how fibulin-5 may act downstream to exert these functional changes, the effect of recombinant human fibulin-5 on ERK activation in Ishikawa cells was assessed. ERK was not phosphorylated at basal levels in Ishikawa cells (Fig. 4A). Recombinant fibulin-5 treatment activated pERK in a dose-dependent manner at 0.1 and $0.5 \mu \mathrm{g} / \mathrm{ml}(\mathrm{P}=0.0440$ and $\mathrm{P}=0.0132$, respectively; $\mathrm{n}=3)$ (Fig. 4A and B).

\section{Discussion}

The current study is the first to identify and determine a functional role for fibulin-5 in endometrial cancer. The findings demonstrated that fibulin-5 gene expression is downregulated in human type I endometrial cancer tissue G1-3 compared with benign endometrium. To determine which cell types express fibulin-5, immunohistochemistry was performed, revealing a shift from epithelial to stromal protein immunolocalisation with increasing tumour grade in women. In vitro, loss of fibulin-5 function promoted Ishikawa endometrial epithelial cancer cell adhesion and proliferation and led to a trend in increased cell invasion and migration, suggesting that loss of fibulin-5 may promote endometrial cancer progression in women. These findings support previous studies in which fibulin-5 was found to be downregulated in other cancer types, including breast, ovarian, colon (9), hepatocellular (10), prostate (11), bladder (12) and lung (13) cancers. However, this contrasts with findings in human fibrosarcoma cells (9) and malignant mammary epithelial cells in breast cancer (14), suggesting that the actions of this ECM protein are tissue-dependent.

The current study is also the first to identify fibulin-5 in the cycling human endometrium. From the present findings, the localisation pattern of fibulin-5 is predominantly cytoplasmic in the glandular epithelium in normal endometrium, with very minimal production in the stroma. In contrast to normal endometrium, fibulin-5 localisation in endometrial cancer tissue transitioned from the endometrial epithelium to the stromal compartment with increasing tumour grade, suggesting a potential role for this protein in EMT. EMT is a critical process in cancer metastasis, during which epithelial cells acquire phenotypes of motile fibroblasts (20). While the functional role of fibulin-5 in endometrial cancer stromal cells remains to be determined, its elevated staining intensity in the tumour stroma with increasing tumour grade suggests it may act to facilitate tumour progression by acting on the local tumour environment (21). In other tumour types, including lung and liver, findings suggest a mechanism in tumour fibroblasts whereby fibulin-5 suppresses metastasis formation by inhibiting production of matrix metalloproteinase 9 and reducing the invasive behavior of fibroblasts (21).

In the present study, fibulin-5 gene silencing resulted in increased Ishikawa cell adhesion. Given the functional role of fibulin-5 as an adhesion molecule via binding of its RGD motif to a number of integrins, including $\alpha 4 \beta 1, \alpha 5 \beta 1, \alpha 9 \beta 1$, $\alpha v \beta 3$, and $\alpha v \beta 3$ (22), this suggests that fibulin-5 may mediate the adhesion phase of invasion via integrin binding. Fibulin-5 knockdown also resulted in increased Ishikawa cell proliferation, supporting previous findings in malignant epithelial cells (9) and suggesting that it may play an antiproliferative role in human endometrial cancer.
A trend in increased Ishikawa cell invasion and migration was also observed. These cells are derived from G1 endometrial cancer, which is not a highly invasive or migratory tumour type (23); this may account for the low baseline levels of invasion and migration, and consequently non-significant functional results in the current study. This model is, however, advantageous for investigating the transition from normal to malignant phenotypes early in endometrial cancer development. To investigate these specific functions, it would therefore be interesting to overexpress fibulin-5 in G2 or G3 cancer cells (otherwise lacking fibulin-5 gene expression). This may allow more precise determination of the effect of fibulin-5 on invasion and migration and could elucidate whether fibulin-5 may have an anti-tumourigenic effect and have any therapeutic potential for endometrial cancers.

To determine what may regulate fibulin-5 expression in human endometrial epithelial cancer cells, Ishikawa cells were exposed to TGF- $\beta$, a cytokine that is known to regulate fibulin-5 in other cell types (14). TGF- $\beta$ upregulated fibulin-5 gene expression in Ishikawa endometrial cancer epithelial cells. Although, this was not investigated at the protein level, it is of interest, given that TGF- $\beta 1$ levels are dramatically downregulated with increasing endometrioid endometrial cancer grade in women, as compared with normal endometrium (24). This could potentially explain, at least partially, the mechanism by which endometrial cancers lose fibulin -5 expression with increasing tumour grade. However, to investigate whether the expression of fibulin- 5 is correlated with the level of TGF- $\beta 1$, it would be of interest to examine the expression of TGF- $\beta 1$ and fibulin-5 in the same cohort of clinical specimens.

Finally, the current study investigated the downstream signaling of fibulin- 5 by examining ERK activation. The ERK signaling pathway is regulated by phosphorylation and dephosphorylation by specific kinases (25). The findings revealed no or low ERK activation at basal levels in Ishikawa cells. By contrast, ERK was activated in a dose-dependent manner in response to fibulin-5, suggesting that it may act functionally via this pathway in this cell type. In support, a previous study demonstrated that fibulin-5 suppressed lung cancer epithelial cell proliferation via activated ERK signalling (9).

In summary, the present study demonstrated that the ECM protein fibulin- 5 is regulated by TGF- $\beta$ in human endometrial epithelial cancer cells and is downregulated in the epithelial compartment in human type I endometrioid endometrial cancer. Functionally, the loss of fibulin-5 gene expression in endometrial epithelial cancer cells enhanced adhesion and proliferation of Ishikawa cells in vitro, potentially mediated by suppressed ERK activation, suggesting that loss of fibulin-5 expression/function could be pro-tumourigenic in women.

\section{Acknowledgements}

The authors would like to acknowledge the support of the Victorian Government's Operational Infrastructure Support Program and the Australian Government National Health and Medical Research Council (NHMRC) Independent Research Institute Infrastructure Support Scheme. Professor Eva Dimitriadis was supported by an NHMRC Fellowship (\#550905). Miss Amy Winship was supported by an Australian Postgraduate Award. 
The authors also wish to acknowledge the technical support of Dr Michelle Van Sinderen (Centre for Reproductive Health, The Hudson Institute of Medical Research, Melbourne, Australia). The authors are grateful to all of the women who donated samples, and to the research nurse, Sister Judi Hocking (Centre for Reproductive Health, The Hudson Institute of Medical Research).

\section{References}

1. Ferlay J, Shin HR, Bray F, Forman D, Mathers C and Parkin DM: Estimates of worldwide burden of cancer in 2008: GLOBOCAN 2008. Int J Cancer 127: 2893-2917, 2010.

2. Soliman PT, Oh JC, Schmeler KM, Sun CC, Slomovitz BM, Gershenson DM, Burke TW and Lu KH: Risk factors for young premenopausal women with endometrial cancer. Obstet Gynecol 105: 575-580, 2005.

3. Elit L and Hirte H: Current status and future innovations of hormonal agents, chemotherapy and investigational agents in endometrial cancer. Curr Opin Obstet Gynecol 14: 67-73, 2002.

4. Di Cristofano A and Ellenson LH: Endometrial Carcinoma. Annu Rev Pathol 2: 57-85, 2007.

5. Pecorelli S: Revised FIGO staging for carcinoma of the vulva, cervix, and endometrium. Int J Gynaecol Obstet 105: 103-104, 2009

6. Albig AR and Schiemann WP: Fibulin-5 function during tumorigenesis. Future Oncol 1: 23-35, 2005.

7. Argraves WS, Greene LM, Cooley MA and Gallagher WM: Fibulins: Physiological and disease perspectives. EMBO Rep 4: 1127-1131, 2003.

8. Yanagisawa H, Schluterman MK and Brekken RA: Fibulin-5, an integrin-binding matricellular protein: Its function in development and disease. J Cell Commun Signal 3: 337-347, 2009.

9. Schiemann WP, Blobe GC, Kalume DE, Pandey A and Lodish HF: Context-specific effects of fibulin-5 (DANCE/EVEC) on cell proliferation, motility, and invasion. Fibulin-5 is induced by transforming growth factor-beta and affects protein kinase cascades. J Biol Chem 277: 27367-27377, 2002.

10. Tu K, Dou C, Zheng X, Li C, Yang W, Yao Y and Liu Q: Fibulin-5 inhibits hepatocellular carcinoma cell migration and invasion by down-regulating matrix metalloproteinase-7 expression. BMC Cancer 14: 938, 2014

11. Wlazlinski A, Engers R, Hoffmann MJ, Hader C, Jung V, Müller M and Schulz WA: Downregulation of several fibulin genes in prostate cancer. Prostate 67: 1770-1780, 2007.

12. Hu Z, Ai Q, Xu H, Ma X, Li HZ, Shi TP, Wang C, Gong DJ and Zhang X: Fibulin-5 is down-regulated in urothelial carcinoma of bladder and inhibits growth and invasion of human bladder cancer cell line 5637. Urol Oncol 29: 430-435, 2011.
13. Yue W, Sun Q, Landreneau R, Wu C, Siegfried JM, Yu J and Zhang L: Fibulin-5 suppresses lung cancer invasion by inhibiting matrix metalloproteinase-7 expression. Cancer Res 69: 6339-6346, 2009.

14. Lee YH, Albig AR, Regner M, Schiemann BJ and Schiemann WP: Fibulin-5 initiates epithelial-mesenchymal transition (EMT) and enhances EMT induced by TGF-beta in mammary epithelial cells via a MMP-dependent mechanism. Carcinogenesis 29: 2243-2251, 2008

15. Yap J, Salamonsen LA, Jobling T, Nicholls PK and Dimitriadis E: Interleukin 11 is upregulated in uterine lavage and endometrial cancer cells in women with endometrial carcinoma. Reprod Biol Endocrinol 8: 63, 2010.

16. Cuman C, Menkhorst EM, Rombauts LJ, Holden S, Webster D, Bilandzic M, Osianlis T and Dimitriadis E: Preimplantation human blastocysts release factors that differentially alter human endometrial epithelial cell adhesion and gene expression relative to IVF success. Hum Reprod 28: 1161-1171, 2013.

17. Livak KJ and Schmittgen TD: Analysis of relative gene expression data using real-time quantitative PCR and the 2(-Delta Delta C(T)) Method. Methods 25: 402-408, 2001.

18. Van Sinderen M, Cuman C, Winship A, Menkhorst E and Dimitriadis E: The chrondroitin sulfate proteoglycan (CSPG4) regulates human trophoblast function. Placenta 34: 907-912, 2013.

19. Lay V, Yap J, Sonderegger S and Dimitriadis E: Interleukin 11 regulates endometrial cancer cell adhesion and migration via STAT3. Int J Oncol 41: 759-764, 2012.

20. Thiery JP, Acloque H, Huang RY and Nieto MA: Epithelial-mesenchymal transitions in development and disease. Cell 139: 871-890, 2009.

21. Møller HD, Ralfkjær U, Cremers N, Frankel M, Pedersen RT, Klingelhöfer J, Yanagisawa H, Grigorian M, Guldberg P, Sleeman J, et al: Role of fibulin-5 in metastatic organ colonization. Mol Cancer Res 9: 553-563, 2011.

22. Nakamura T, Lozano PR, Ikeda Y, Iwanaga Y, Hinek A, Minamisawa S, Cheng CF, Kobuke K, Dalton N, Takada Y, et al: Fibulin-5/DANCE is essential for elastogenesis in vivo. Nature 415: 171-175, 2002.

23. Meng YG, Han WD, Zhao YL, Huang K, Si YL, Wu ZQ and $\mathrm{Mu}$ YM: Induction of the LRP16 gene by estrogen promotes the invasive growth of Ishikawa human endometrial cancer cells through the downregulation of E-cadherin. Cell Res 17: 869-880, 2007.

24. Perlino E, Loverro G, Maiorano E, Giannini T, Cazzolla A, Napoli A, Fiore MG, Ricco R, Marra E and Selvaggi L: Down-regulated expression of transforming growth factor beta 1 mRNA in endometrial carcinoma. Br J Cancer 77: 1260-1266, 1998.

25. Shaul YD and Seger R: The MEK/ERK cascade: From signaling specificity to diverse functions. Biochim Biophys Acta 1773: 1213-1226, 2007. 